Article

\title{
Understanding Mothers' Infant Feeding Decisions and Practices
}

\author{
Emma Head
}

School of Social Science and Public Policy, Keele University, Staffordshire ST5 5BG, UK; e.l.head@keele.ac.uk

Academic Editor: Martin J. Bull

Received: 13 December 2016; Accepted: 27 April 2017; Published: 17 May 2017

\begin{abstract}
This paper begins with a discussion of social research which seeks to critique the emphasis on breastfeeding in infant feeding health promotion. The key themes of this research center on science, risk, and morality but other factors can also shape mothers' decisions and practices regarding infant feeding, and particularly, breastfeeding. The paper explores a range of research studies which together highlight the wide range of social, cultural, and economic factors implicated in infant feeding decisions and practices. The discussions here demonstrate that social and economic factors, familial and social networks, interactions with health professionals, cultural contexts which sexualize women's bodies, and experiences of public space, can all play a role in shaping how mothers negotiate infant feeding. This broad conceptualization of the factors that shape infant feeding practices offered by social research poses a challenge to the more simplistic accounts of infant feeding decisions implicit in public health promotion. It also demonstrates the profoundly social quality of infant feeding decisions that women make and the particular contributions that social research can make to our understandings of this area.
\end{abstract}

Keywords: infant feeding; breastfeeding; motherhood; childhood; risk

\section{Introduction}

In recent years some social research on infant feeding has developed a critique of public health agendas based on the promotion of breastfeeding. The aims of health promotion around infant feeding in Britain and in other Western countries have been to increase both initiation rates and the duration of breastfeeding by mothers. Social research has examined the impact of this agenda on women's self-identities and experiences of motherhood and sought to analyze what this tells us about parenting culture more generally. The first part of this paper discusses the development of social research that focuses on the promotion of breastfeeding in public health campaigns and by health professionals. I argue that this work tends to conceptualize the choices of women as constrained by overbearing public health messages which need to be 'rolled back' to enable women to make feeding decisions according to their own wishes. However, other scholarship on infant feeding has explored wider cultural, social, and economic and factors which also influence women's ideas and practices around infant feeding. These factors are explored in the second part of this paper.

In the concluding section, I argue that social research on infant feeding needs to continue to resist the regulation of women's 'choices' and support women's autonomy, whilst exploring the wider social, cultural, and economic factors that shape childrearing practices. Social research on infant feeding has demonstrated the profoundly social nature of decisions which in public health terms might be viewed as more straightforwardly, natural. The broad exploration of infant feeding found in social research —one that recognizes its temporal, embodied, and emotional dimensions and that it takes place in the wider context of specific health and policy regimes-is thus a challenge for more simplistic accounts which focus on changing attitudes or behaviors in an individualist way. 


\section{Morality, Risk, and Science in Infant Feeding Research}

In recent years researchers have critically examined the impact that privileging breastfeeding in health promotion has on women's experiences of motherhood (Lee 2011a, p. 1058). Lee (2011a) asserts that before 2000, the most notable study in this area was Carter's work on Feminism, breasts and breast-feeding (Carter 1995). Lee notes this was followed up by a series of papers from Murphy $(1999,2003)$. Murphy's work (Murphy 2003) is based on longitudinal qualitative work with 36 women during pregnancy and in the first year of their infant's life. Murphy argues that infant feeding choices are complex. She explores the "accountability talk" in the narratives of her interviewees, showing that mothers "can be seen to be constructing an image of themselves as moral members of society" in these accounts (Murphy 1999, p. 191). The idea that infant feeding becomes moralized in contexts where policy promotes breastfeeding is bound up with the notions of identity, a recurrent theme in infant feeding research. Murphy's wider program of research connected women's experiences of infant feeding with "wider sociologies of risk, public health, and identity" (Lee 2011a, p. 1059). These themes have continued to be explored by scholars associated with Parenting Culture Studies (see (Lee et al. 2014)) who consider what the emphasis on breastfeeding in health promotion tells us about wider society and the culture of parenting (Lee 2011a, p. 1059).

Since the 1990s, the significance of risk in the social construction of parenting and childhood has been a concern of researchers interested in cultures of parenting. This is perhaps indicative of the wider hold that ideas of risk and security had on sociological imaginations at this time, following the publication of Giddens' The Consequences of Modernity (Giddens 1990) and Beck's Risk society (Beck 1992). A key work here is Furedi's Paranoid Parenting (Furedi 2001) which identifies a culture of paranoia around the actions of parents. This is said to shape the interactions of parents and children and shape how parents relate to other adults in society—relationships become marked by fear and mistrust. Writers in the field of Parenting Culture Studies argue that over the last twenty years or so, the task of childrearing has expanded, meaning parents are now expected to fulfil a wider range of tasks than in the past (Lee et al. 2010, p. 294). Significantly, this expansion of parenting has happened “ ... in tandem with the development of the notion that children are 'at risk'", and, consequently, parents become constructed as "(inadequate) risk managers" (Lee et al. 2010). Risk has been a particularly salient idea for understanding how women experience infant feeding (for example, (Murphy 1999; Faircloth 2010; Lee 2007a; 2007b)). This is in line with the 'breast is best' message rooted in scientific claims that formula milk is more 'risky' for infants than breastmilk. Leaflets and websites promoting breastfeeding tend to emphasize the health benefits of breastmilk over formula milk or mixed feeding (for example, (NHS 2016)). Researchers have thus argued that the promotion of breastmilk as 'better than' formula milk has informed a moralized discourse around infant feeding where the 'good mothers' are those who breastfeed their infants (Lee 2011b).

\subsection{Scientific Knowledge and Infant Feeding}

Critiques of the way that ideas of risk and science have dominated debates around infant feeding are based around two main arguments. The first of these questions the validity of the scientific evidence that supports the promotion of breastfeeding. Following on from this, the second is concerned with the restrictions placed on women's abilities to make meaningful choices around infant feeding that the current formulation of health promotion supports. Joan Wolf calls into question the credibility of scientific evidence that finds breastmilk significantly superior to formula milk as a way to nourish infants (Wolf 2007; 2011). Wolf (2007), writing in a North American context, casts doubt on scientific research which claims to demonstrate a causal connection between breastfeeding and a range of positive health, developmental, and social outcomes in childhood and into adulthood. Wolf's research carefully details the difficulties of conducting robust epidemiological research on infant feeding. She argues that breastfeeding " ... cannot be distinguished from the decisions to breast-feed, which, irrespective of socioeconomic status or education, could represent an orientation toward parenting that is itself likely to have a positive impact on children's health" (Wolf 2007, p. 602). 
The identification of weaknesses with knowledge claims about the superiority of breastmilk form part of the explanation for Wolf's critique of the way breastfeeding campaigns in the USA have been run. Wolf argues that the National Breastfeeding Awareness Campaign (2004-2006) was misguided as it was based on inconsistent research, "capitalized on public misunderstanding of risk and risk assessment", and "exploited deep-seated normative assumptions about the responsibility that mothers have to protect babies and children from harm ..." (Wolf 2007, p. 596). Wolf (2007, p. 617) suggests that breastfeeding is central to the discourse of "total motherhood" which is synonymous with ideas of "intensive mothering" (see (Hays 1996)) and "the new momism" (see (Douglas and Michaels 2004)). Total motherhood demands that mothers become experts in a number of areas, including safety; that they protect their children from threats (or possible threats) to their development; and that they promote the optimal developmental of their child (Wolf 2007, p. 615). 'Total motherhood' entails an orientation to risk where 'good mothering' reduces any risk to infants, regardless of how small the likelihood of danger and of the potential costs to mothers (Wolf 2007, p. 615). In terms of infant feeding, this discourse of 'total motherhood' means that feeding decisions are understood as constrained by scientific evidence which overstates the riskiness of formula milk in a wider cultural context where mothers are expected to avoid any possible risks to their infants.

\subsection{Promoting Breastfeeding and Understanding Women's Choices}

This concern about the ability of women to make meaningful choices around infant feeding is a key feature of research which critiques the promotion of breastfeeding on health grounds. Lee (2011b, p. 7) writes that the promotion of breastfeeding on scientific grounds can be understood " ... as the outcome of a cultural process in which the authority of science and medicine is borrowed by lobbyists and campaigners", resulting in the diminishing of other forms of authority and the "de-authorization" of the parent. Knaak's research (Knaak 2005), a textual analysis of a selection of Dr. Spock childcare manuals published in Canada from 1946 to 1998, makes an interesting contribution to these debates on the implications of particular constructions of breastfeeding. She argues that over time the constraints on choice around infant feeding have increased so the presentation of breastfeeding has become more of a directive than a choice. Knaak (2005, p. 211) identifies how the 'choice' to breastfeed has become increasingly pressured as it is not just physical health outcomes that are linked to breastfeeding but "increasingly strong interconnections" are made with mother-infant bonding. This adds an additional pressure for women negotiating infant feeding and means their choices are constrained because formula feeding is represented negatively (Lee 2011b, p. 8).

A useful discussion of the complexity of thinking about 'choice' in relation to infant feeding is found in research by Murphy et al. (1998). This is qualitative longitudinal research with mothers and develops a sociological approach to feeding practices (Murphy et al. 1998). Murphy et al. caution against an overly simplistic and deterministic approach to understanding feeding practices. They argue that a focus on 'choice' can mean a neglect of the "processual nature of such decisions and the way in which they are made and remade over time" (Murphy et al. 1998, p. 265). Murphy et al. also identify the problem with defining actions as choices, as one which can mask wider constraining factors:

"We are arguing that it is misleading to treat women's feeding practices as the simple expression of individualistic preferences. Rather, such practices reflect the material and sociocultural contexts in which their decisions are made. The risk of defining such decisions as choice is that we camouflage the constraints under which women deliberate and act". (Murphy et al. 1998, pp. 262-63)

The significance of Murphy et al.'s work here is in recognizing the complexity of infant feeding decisions and emphasizing the significance of wider social, material and cultural forces at play. This issue is partly recognized by some authors whose work is critical of the promotion of breastfeeding, for example, Lee (2007a, p. 1087) notes that infant feeding should be studied alongside an appreciation of other aspects of maternal experience. Elsewhere, Lee tackles the issue of choice noting that this 
term, " ... fails to capture women's real experience" because choices are not just made in the abstract, but, " . . mothers make pragmatic decisions in a response to their experience" (Lee 2007b, p. 306). The next sections of this paper consider the range of social, cultural, and economic factors that shape infant feeding decisions and practices. This demonstrates the breadth of social research on infant feeding. These discussions sketch out what forms the constraints that Murphy et al. refer to might take and potentially shape women's perceptions, decisions and experiences of infant feeding. This discussion begins with a consideration of the statistical trends in infant feeding which demonstrates a social patterning to mothers' decisions.

\section{Social Trends and Economic Factors}

Infant feeding research has critiqued public health campaigns on infant feeding but the extent to which these campaigns have influenced women's feeding decisions is difficult to assess. In the UK, quantitative data on infant feeding has been collected at five year intervals by the Infant Feeding Survey (IFS) since 1975. The most recent survey was conducted in 2010 as the 2015 survey was cancelled. The IFS data shows that whilst rates of breastfeeding initiation have increased over time, the duration that most women exclusively breastfeed for is significantly out of step with public health advice (McAndrew et al. 2012a). Sixty-nine percent of mothers exclusively breastfed their infants at birth, falling to $46 \%$ at the one week mark; $23 \%$ at six weeks; and one percent at six months (McAndrew et al. 2012a). Mothers in the 2010 survey were breastfeeding for longer than in 2005 (this includes mixed feeding), and more women initiated breastfeeding than had previously been reported, 81\% in 2010, 76\% in 2005 (McAndrew et al. 2012a). McAndrew et al. suggest that policy developments for improving support and information to mothers about breastfeeding " ... may have had an impact" (McAndrew et al. 2012a). This impact on behavior is perhaps more muted than might be expected given the strong emphasis on the benefits of breastfeeding in public health information.

Rates of breastfeeding vary considerably between different countries and between women in the same societies who have different social characteristics. This suggests that breastfeeding rates can be understood as influenced in part by sociocultural factors and not simply as the result of biological or physiological processes. The Infant feeding survey (McAndrew et al. 2012a; 2010b) identifies different rates of breastfeeding amongst women based on particular characteristics. For example, mothers aged over 30; women from particular minority ethnic groups; and women who were in professional or managerial occupations, all had breastfeeding rates that were between six and 16 percent higher than the average rate (McAndrew et al. 2012a, p. 1). Data from the IFS also shows the connections between the feeding practices of mothers and their family histories and suggested friendship networks were significant. Twenty-nine percent of breastfeeding mothers who themselves had been exclusively formula fed stopped breastfeeding within two weeks of their infant's birth. In contrast, this was the case for $9 \%$ of the breastfeeding mothers who had been breastfed (McAndrew et al. 2012a, p. 6). Twenty-six percent of women who were breastfeeding and reported that most of their friends only formula fed their infants stopped breastfeeding in the first two weeks. In comparison, $6 \%$ of breastfeeding mothers who reported that most of their friends breastfed stopped breastfeeding in the same time period (McAndrew et al. 2012a, p. 6). These findings suggest a connection between friendship and familial networks and the duration or persistence of breastfeeding. This recalls Murphy et al.'s observation about the 'processual' nature of infant feeding decisions (Murphy et al. 1998). The IFS findings also call attention to the importance of what have been termed 'local maternal cultures' (see, for example, (Thomson 2008)): the idea that certain values, beliefs and norms about motherhood are shared in particular localities. It may be the case that strong locally held norms about the appropriateness of formula milk and what is best for infants actually discourages breastfeeding for some women and enables them to resist public health discourses on infant feeding.

Some research has explored the complex ways in which the social and economic positioning of women in high-income countries shapes the rates of breastfeeding (Frank 2015). In Frank (2015) Canadian study of twenty mothers in low-income households, the impact of severe food insecurity on 
women's own food consumption and on the success of breastfeeding is explored. Frank found it was women who lived in the most food insecure household who were least likely to initiate breastfeeding or who had shorter durations of breastfeeding. Frank notes that feeding practices are shaped by the "social conditions of poverty" (Frank 2015, p. 203). The significance of the "cultural and material realities" which make breastfeeding difficult are also a feature of Wall's research on Canadian health education materials (Wall 2001, p. 605). Wall argues that these wider factors are constructed in breastfeeding promotion materials as individual problems for women to solve with the assistance of health professionals. In addition to material factors shaping feeding decisions, Rippeyoung and Noonan (2012) have identified a personal economic cost of longer duration breastfeeding for women in the US. These findings gives weight to Rippeyoung's earlier work where she suggested state promotion of breastfeeding should be viewed with suspicion; identifying the promotion of breastfeeding as an individual responsibility as a strategy for the state to avoid the responsibility for tackling social problems (Rippeyoung 2009).

It might be that emphasizing breastfeeding has had more impact on shaping the feelings of women about infant feeding than promoting behavioral change. Lee notes that most women in Britain will feed their babies with formula milk and a large minority of her survey respondents felt guilt and concern about this (Lee 2007b, p. 296). There is an increasing distance between official discourses of infant feeding and the everyday experiences of mothers (Lee 2011a). Lee suggests that as breastfeeding has been constructed as an integral part of 'good' mothering for some women, the use of formula milk is understood as a failure, rather than a pragmatic action (Lee 2011a, p. 1066). Similarly, Taylor and Ebert (2012) have argued that women's feelings when breastfeeding is not successful are connected to the failure to meet idealized notions of motherhood. The gap between public health promotion and women's experiences adds weight to the recommendation that those promoting breastfeeding should try to roll back the moralization of infant feeding and, more broadly, that the politicization of parenting needs to be undone (Lee et al. 2010). Another implication of these observations is that factors beyond state intervention and the promotion of breastfeeding are also important in shaping the way mothers negotiate infant feeding decisions and practices. These factors include social and familial networks, the interactions between mothers and health professionals, embodiment, and experiences of public space. The focus of this paper now turns to these concerns.

\section{Family Relationships, Social Networks, and Infant Feeding}

A number of studies suggest social networks influence orientations to and experiences of infant feeding. Lavender et al. (2006) explored the perceptions and experiences of breastfeeding in a family context, noting that existing research on social networks was through the gaze of the mother. Lavender et al. encountered difficulties in recruiting family members to their research project which they speculate may have been connected to feelings of embarrassment about the topic. Nevertheless, there were some useful findings particularly the idea of "disparate communications"; the difficulties and differences in talking about infant feeding that were reported between mothers and other family members (Lavender et al. 2006, p. 152). Family members were reluctant to talk about finding breastfeeding personally embarrassing but raised this as a feeling that other people might have. There was a sense that breastfeeding did cause difficulties in interpersonal relationships for some of the mothers in the study and they reported a lack of practical and emotional help that could feel isolating. For example, it was noted that family members might leave the room when breastfeeding was taking place (Lavender et al. 2006, p. 152).

Lavender et al.'s small scale study could help us to understand why the move from breastfeeding to formula milk (or a mixed feed approach), can be seen as having the "pragmatic advantages" for women that Lee reports (Lee 2007a, p. 1080). In a study that mixed qualitative and quantitative approaches, $76 \%$ of mothers using formula agreed that they were "'pleased to find a solution that made things easier'"' (Lee 2007b, p. 300). This experience of the ease of formula milk was explored in the qualitative interview phase of this project and one dimension of the ease of formula milk was that 
other family members could help. It is plausible that at a time when parenting culture is shaped by an ideology of child-centeredness (see (Knaak 2005)), it might be easier for mothers to marshal help that involves direct involvement with an infant, i.e., bottle feeding, than in the case of breastfeeding, where help is indirect and might need to be given in the form of taking care of the mother. Along similar lines to this observation, Trickey and Newburn suggest new priorities in the areas of infant feeding and support, including a potential role for ante-natal education that raises awareness "... among women and their partners of the need for practical domestic help, emotional encouragement, and of how to access prompt skilled support ... " More broadly, these recommendations suggest the need for emphasis to be placed on the care of new mothers, not solely on their infants. This opens up the possibility of thinking about how to recast infant feeding support and advice to dispense with the unhelpful moralization of infant feeding to support the decisions that women want to make (Trickey and Newburn 2012, p. 89).

Social networks shape infant feeding decisions and seem to prompt a warming to, or cooling off from, breastfeeding for new mothers. This can sometimes be connected to a clash between the dominant discourses of what a 'good mother' does, exclusively breastfeed her infant, and what more locally held understandings might be. Research that is critical of breastfeeding promotion tends to imply that infant feeding is inevitably an identity project for most new mothers and this is a finding that emerges from some qualitative studies. For example, Lee et al. (2010, p. 296) characterizes the work of Faircloth (2010) and Knaak (2010) as showing how "maternal identity is formed strongly in relation to culturally-validated notions regarding the superiority of breastfeeding". Faircloth (2010, p. 360) observes that whilst formula feeding is statistically a norm, mothers who nourish their infants with formula have to "engage in 'identity work' to create themselves as moral citizens in the face of 'deviance'". Faircloth's research is based on the experiences of women who breastfeed their children to 'full term' when weaning from the breast is child-led and can often continue past infancy and toddlerhood (Faircloth 2009, p. 63). Faircloth identifies that these women engage in complex identity work as their long-term feeding marginalizes them in terms of "statistical, ideological and cultural norms" (Faircloth 2010, p. 357). These findings again show that the complexities around infant feeding practices and the difficulties that women have in sustaining their self-identities as 'good' mothers.

\section{Infant Feeding and the Role of Health Professionals}

Some research suggests versions of 'good motherhood' adapt, resist, or ignore public health discourses that connect the idea of the 'good mother' with breastfeeding. Health professionals can be part of a group who influence the way in which mothers think about infant feeding and 'good motherhood'. Marshall et al. (2007) research, based on observations between mothers and health professionals and qualitative interviews, found their participants did not connect breastfeeding and 'good mothering' in a straightforward way. Instead, this idea was confirmed or called into question by perceptions of their infant. Marshall et al. (2007) note that the idea of breastfeeding as 'good mothering' was maintained when the infant was healthy and content but undermined if the infant was feeding frequently without apparent weight gain, or if he/she was unsettled. Marshall et al. make the important observation that 'breast is best' is one discourse around infant feeding and "... women are exposed to a considerably more diverse of set of values and influences across their social networks" (Marshall et al. 2007, p. 2158). Therefore, women may encounter and negotiate different ideas of what good motherhood entails and this is not necessarily determined by public health messages. The recognition of the complexities of how health promotion messages are translated at the local level is a key finding of studies that incorporate observational research into their methods and place emphasis on the importance of space and place in the conceptual schema of their projects (for example, (Mahon-Daly and Andrews 2002; Dykes 2005)).

Mahon-Daly and Andrews' research (Mahon-Daly and Andrews 2002) is based on observations of a health visitor run mother and baby group and qualitative interviews with mothers who attended. A total of 27 women, mostly middle class, were observed at the mother and baby group. All of 
the women had initiated breastfeeding and in the course of the study around half of the women ceased breastfeeding or began to mix feed by introducing formula milk. From their observational research, Mahon-Daly and Andrews argue that one dimension of the mother and baby group was that it functioned as a medical space, where babies were weighed and assessed; this meant it ". . s somewhat medicalized and objectified breastfeeding" (Mahon-Daly and Andrews 2002, p. 67). In interactions between health professionals and mothers, bottle-feeding was sometimes favored over breastfeeding and sometimes this resulted in mothers renegotiating breastfeeding decisions (Mahon-Daly and Andrews 2002). Mahon-Daly and Andrews argue that this medicalization of breastfeeding "may well have contributed to the early cessation of breastfeeding, or the initiation of mixed feeding" (Mahon-Daly and Andrews 2002, p. 68). This study suggests that social interactions, with health professionals in this case, can influence feeding decisions and undermine breastfeeding. Interactions between health professionals and mothers do not always simplistically reproduce the 'breast is best' message. For example, Marshall et al. describe how one of their participants who was frequently breastfeeding her infant was advised by a health visitor to introduce formula at night (Marshall et al. 2007, p. 2154). These studies point to the importance of observational research that can capture the understandings of infant feeding developed in specific local contexts. At the local level understandings of infant feeding can be produced between health professionals and mothers that challenge the dominant ideas of health promotion and shape mothers' feeding decisions.

Interactions between health professionals and mothers were a central concern of Dykes' critical ethnographic study of two English maternity wards (Dykes 2005). Dykes observed interactions between midwives and postnatal women and interviewed both in groups. The argument that Dykes develops has parallels with Mahon-Daly and Andrews' observation about the medicalization of breastfeeding (Mahon-Daly and Andrews 2002). Dykes charts how mothers' early experiences of infant feeding were shaped by dominant Western understandings of the body, where bodies are viewed mechanistically, and the female body is seen as "inadequate and potentially defective" (Dykes 2005, p. 2290). Dykes reports that a "striking" finding of her research was women's "lack of confidence and trust in their ability to produce enough milk or milk of the right quality"; significantly, this lack of confidence was mirrored in the language and actions of many midwives (Dykes 2005, p. 2287). Women used objectifying language when talking about their bodies and breasts and conceptualized their bodies as apart from themselves. This understanding of breastfeeding body was reinforced by the mechanistic language adopted by the midwives studied. Dykes makes a comparison between this understanding of the breastfeeding body and the industrial metaphor that other work has used to conceptualize the laboring body as a site of production in hospital settings (see (Martin 1987)). Dykes suggests that infant feeding promotion uses a mechanistic conceptualization of breastfeeding which emphasizes the qualities of breastmilk as a product. She advocates that this understanding of breastfeeding should be balanced by stressing ideas of relationality so that breastfeeding is constructed as part of the relationship between mother and child, not solely the exchange of a product with nutritional benefits (Dykes 2005, p. 2291).

The kind of insights offered by Mahon-Daly and Andrews, Marshall et al., and Dykes, show the importance of conducting infant feeding research in healthcare settings and suggest the complexities of how infant feeding messages are transmitted and negotiated in interactions between mothers and medical professionals. It does not appear to be the case that public health messages are passed from health professionals to mothers in a straightforward way. Dykes' works also brings into focus the usefulness of analyzing breastfeeding as an activity that happens in place and that is an embodied experience. Women's perceptions of breastfeeding are connected to their understandings of bodies in a general sense and their feelings about their own body, in particular. An exploration of these meanings of the body is a significant strand of infant feeding research and shows the importance of exploring wider cultural ideas about women's bodies in the developing understanding of infant feeding practices. 


\section{The Body and Infant Feeding}

A number of feminist writers have argued that contemporary society is marked by an increasing objectification of women's bodies and a hyper-sexualization of women's bodies in particular forms of popular culture (see (Bradley 2013)). Some campaigning by feminist groups has centered on the representations of women's bodies, for example, the No More Page 3 campaign aimed at The Sun newspaper in the UK. This campaign, launched in 2012, was against the use of 'topless' models in a regular feature in the newspaper. The appropriate female body shape is narrowly defined in media images and women are encouraged to see their bodies as dysfunctional and in need of modification and improvement. This cultural objectification of women's bodies and sexualization of breasts (Millsted and Frith 2003) means that pregnant women and new mothers hold particular understandings of their own bodies and of the appropriateness of breastfeeding before they begin to decide how their infant will be fed.

Some scholarship on breastfeeding has sought to understand how women's perceptions and experiences of breastfeeding are shaped by cultural meanings around bodies and the fact that breastfeeding is an embodied process. Bartlett (2002) takes up Grosz's call for a 'corporeal feminism' (Grosz 1994) and applies this to breastfeeding. Bartlett's work is a challenge to understandings of breastfeeding which are biologically based, as she argues this narrative is incomplete without an understanding of women's subjectivity. She contends that "... historical, cultural and environmental social relations impact on breastfeeding operations in quite unpredictable biological ways via hormonal activity, especially the release of oxytocin" (Bartlett 2002, p. 375). Here, Bartlett is moving beyond a suggestion that cultural meanings around bodies might shape infant feeding 'decisions' to a suggestion that breastfeeding as a physiological process is influenced by the wider social and cultural context. Bartlett claims that " ... a woman's lived experiences are crucial to her body's lactational responses" (Bartlett 2002, p. 375). This argument resonates with themes from Mahon-Daly and Andrews' (Mahon-Daly and Andrews 2002, p. 63) discussion of insufficient milk syndrome (IMS), which refers to the inability of a mother to adequately produce breastmilk or a mother's perception that this is the case. They argue that IMS is found in contexts where breastfeeding is marginalized or is not supported and that an understanding of IMS is developing that conceptualizes it as shaped by "physiological or nutritional practices" and "very specific local, social and family contexts" (Mahon-Daly and Andrews 2002, p. 63). If these observations about women's bodies and the sexualization of popular culture are taken into account, it becomes unsurprising that women can feel uncertain about breastfeeding, and, as Dykes (2005) found can lack confidence in their ability to produce milk in sufficient quantity or quality for their infant. Social research is this area suggests the importance of understanding women's experiences of their bodies into infant feeding research and the complexity of the relationships between biological and social processes.

\section{The Role of Public Space}

The importance of recognizing breastfeeding as an embodied experience and as one that can be shaped by wider cultural and social ideas becomes particularly clear when research on infant feeding and space is considered. Many women who consider breastfeeding their infant will reflect on how they will feed their baby when outside of the home. Research from a range of perspectives acknowledges the difficulties women can experience when breastfeeding in public spaces. In Murphy's (Murphy 1999, p.202) analysis of mothers' talk about breastfeeding, she identifies how women appeal to ideals of modesty and discretion in feeding and she relates this to the link between breasts and sexuality in Western culture. Murphy argues that mothers' constructions of breastfeeding as not "rule breaking" is partly a defense against the possibility of others viewing them as "brazen women" (Murphy 1999, pp. 202, 204). Here, the dominant understanding of women's breasts as having a primarily sexual function impacts on perceptions and experiences of infant feeding.

The IFS 2010 asked mothers about feeding their infants outside of the home. The survey found that breastfeeding outside of the home was more likely to be undertaken by particular mothers: those who 
were reporting on their second (or later) baby; women who were older; in the least deprived quintile on socio-economic status; or in managerial/professional occupation. Eleven percent of the women who had breastfed in public reported they had "been stopped or been made to feel uncomfortable doing so" (McAndrew et al. 2012a, p. 10). In Boyer's mixed methods research on breastfeeding outside the home (on a much smaller scale than the IFS research), $49 \%$ of participants reported a negative experience while breastfeeding in public (Boyer 2012, p. 556). Boyer adapts Ahmed's work (see (Ahmed 2010)) on the figure of the killjoy and her impact on public comfort to explain why women face disapproval for breastfeeding in public spaces. In these terms, a breastfeeding infant signals a body 'out of place'; breastfeeding in the public realm disrupts the comfort of others. Boyer's work suggests that breastfeeding in public challenges gendered norms around the appropriate use of space as breastfeeding brings both care-work and sexualized bodies into public view and public space (Boyer 2012, p. 556). The significance of public spaces in shaping understandings of breastfeeding has led to calls for breastfeeding to be seen as part of intimate citizenship (Smyth 2008). Social research here calls attention to the wider sociocultural meanings around women's bodies and public space and the potential of these factors to shape women's infant decisions and practices.

\section{Conclusions: Social Research and Infant Feeding}

This paper has attempted to bring together a range of studies on infant feeding to demonstrate the breadth and significance of social research in this area. This research encompasses studies which critically engage with health promotion messages and research which explores the wider social, cultural and economic factors which shape mothers' feeding practices. Infant feeding practices need to be understood as taking place in particular familial and social networks, in particular spaces, between bodies, and shaped by interactions between mothers and health professionals. This assertion is in a similar vein to Avishai's observation that "women approach breastfeeding within a specific social, cultural, political and historical context, with the understanding that breastfeeding is always a cultural event shaped by specific institutional, historical, cultural, raced, gendered, and sexualized arrangements". Social research on infant feeding underlines the wide range of factors that can influence infant feeding decisions and shows the importance of developing a more complex analysis of these factors than public health focused accounts tend to allow (Avishai 2007, p. 150).

Social researchers face particular challenges in developing the scholarship of infant feeding. One of these is recognizing the agency women have in making infant feeding decisions, whilst at the same time understanding and exploring the wider cultural and social processes that shape these 'decisions'. It is important to recognize how social class, age, and ethnicity can position women differently in relation to a range of factors that potentially shape infant feeding practices. For example, Groleau et al. (2013) in research with low-income French-Canadian women, argue that poorer women are less likely to be able to resist dominant cultural norms which "hypersexualize" breasts and sexualize breastfeeding. The diversity amongst women and their differential access to power and resources could more heavily inform infant feeding research agendas. This might necessitate reflection on the range of research methods used in empirical work, and the usefulness of observation methods has been explored in this paper.

Some differences in research on infant feeding relate to the disciplines researchers are working in and to the kind of social change their research agendas are orientated to. Some researchers conclude there is little role for social policy or health promotion in the realm of infant feeding. For other researchers, there is a sense that the state does have a role but that this needs to be refocused, to be moved away from an emphasis on breastfeeding. Dykes (2005) advocates a shift from the emphasis on the nutritional qualities of breastmilk and the productive nature of breastfeeding to a focus on the relationality of infant feeding. Trickey and Newburn (2012) suggest ways in which antenatal education could be improved, this might include work with partners of pregnant women. Similarly, Groleau et al. note that public health measures on breastfeeding could include family members and friends of pregnant women (Groleau et al. 2013, p. 258). They also suggest that dominant representations of 
women's bodies need to be challenged so that the "nutritional role of breast" is visible in public spaces and in the media (Groleau et al. 2013). The discussions in this paper would suggest that the goals of infant feeding research need to include further development of an understanding of how infant feeding practices are shaped by a range of personal, social, cultural and economic factors, whilst holding onto a commitment to enabling women to make meaningful choices for themselves and their infants.

Conflicts of Interest: The author declares no conflict of interest.

\section{References}

Ahmed, Sara. 2010. Killing joy: Feminism and the history of happiness. Signs 35: 571-94. [CrossRef]

Avishai, Orit. 2007. Managing the lactating body: The breast-feeding project and privileged motherhood. Qualitative Sociology 30: 135-52. [CrossRef]

Bartlett, Alison. 2002. Breastfeeding as headwork: Corporeal feminism and meanings for breastfeeding. Women's Studies International Forum 25: 373-82. [CrossRef]

Beck, Ulrich. 1992. Risk Society. London: Sage Publications.

Boyer, Kate. 2012. Affect, corporeality and the limits of belonging: Breastfeeding in public in the contemporary UK. Health \& Place 18: 552-60.

Bradley, Harriet. 2013. Gender, 2nd ed. Cambridge: Polity Press.

Carter, Pam. 1995. Feminism, Breasts and Breast Feeding. New York: St. Martin's Press.

Douglas, Susan J., and Meredith W. Michaels. 2004. The Mommy Myth. New York: Free Press.

Dykes, Fiona. 2005. 'Supply' and 'demand': Breastfeeding as labour. Social Science \& Medicine 60: 2283-93.

Faircloth, Charlotte. 2009. 'Culture means nothing to me': Thoughts on nature/culture in narratives of long-term breastfeeding. Cambridge Anthropology 28: 63-84.

Faircloth, Charlotte. 2010. 'If they want to risk the health and well-being of their child, that's up to them': Long-term breastfeeding, risk and maternal identity. Health, Risk \& Society 12: 357-67.

Frank, Lesley. 2015. Exploring infant feeding practices in food insecure households: What is the real issue? Food and Foodways 23: 186-200. [CrossRef]

Furedi, Frank. 2001. Paranoid Parenting. London: Allen Lane.

Giddens, Anthony. 1990. The Consequences of Modernity. Stanford: Stanford University Press.

Groleau, Danielle, Catherine Sigouin, and Nicole Anne D'souza. 2013. Power to negotiate spatial barriers to breastfeeding in a Western context: When motherhood meets poverty. Health E Place 24: 250-59.

Grosz, Elizabeth. 1994. Volatile Bodies. Bloomington: Indiana University Press.

Hays, Sharon. 1996. The Cultural Contradictions of Motherhood. New Haven: Yale University Press.

Knaak, Stephanie. 2005. Breast-feeding, bottle-feeding and Dr. Spock: The shifting context of choice. Canadian Review of Sociology/Revue Canadienne De Sociologie 42: 197-216. [CrossRef]

Knaak, Stephanie. 2010. Contextualising risk, constructing choice: Breastfeeding and good mothering in risk society. Health, Risk $\mathcal{E}$ Society 12: 345-55.

Lavender, Tina, Catherine McFadden, and Lisa Baker. 2006. Breastfeeding and family life. Maternal and Child Nutrition 2: 145-55. [CrossRef] [PubMed]

Lee, Ellie. 2007a. Health, morality, and infant feeding: British mothers' experiences of formula milk use in the early weeks. Sociology of Health $\mathcal{E}$ Illness 29: 1075-90.

Lee, Ellie. 2007b. Infant feeding in risk society. Health, Risk \& Society 9: 295-309.

Lee, Ellie. 2011a. Breast-feeding advocacy, risk society and health moralism: A decade's scholarship. Sociology Compass 5: 1058-69. [CrossRef]

Lee, Ellie. 2011b. Feeding babies and the problem of policy. CPCS Briefing, University of Kent. Available online: https://blogs.kent.ac.uk/parentingculturestudies/files/2011/02/CPCS-Briefing-onfeeding-babies-FINAL-revised1.pdf2011 (accessed on 9 December 2016).

Lee, Ellie, Jan Macvarish, and Jennie Bristow. 2010. Risk, health and parenting culture. Health, Risk \& Society 12: 293-300.

Lee, Ellie, Jennie Bristow, Charlotte Faircloth, and Jan Macvarish. 2014. Parenting Culture Studies. Basingstoke: Palgrave Macmillan. 
Mahon-Daly, Patricia, and Gavin J. Andrews. 2002. "Liminality and breastfeeding: Women negotiating space and two bodies". Health \& Place 8: 61-76.

Marshall, Joyce, Mary Godfrey, and Mary J. Renfrew. 2007. Being a 'good mother': Managing breastfeeding and merging identities. Social Science \& Medicine 65: 2147-59.

Martin, Emily. 1987. The Woman in the Body. Boston: Beacon Press.

McAndrew, Fiona, Jane Thompson, Lydia Fellows, Alice Large, Mark Speed, and Mary J. Renfrew. 2012a. Infant Feeding Survey 2010: Summary. Health and Social Care Information Centre. Available online: http:/ / www.hscic.gov.uk/catalogue/PUB08694/ifs-uk-2010-sum.pdf (accessed on 9 December 2016).

McAndrew, Fiona, Jane Thompson, Lydia Fellows, Alice Large, Mark Speed, and Mary J. Renfrew. $2010 \mathrm{~b}$. Infant Feeding Survey 2010: Chapter Two-Incidence, Prevalence and Duration of Breastfeeding. Available online: http://www.hscic.gov.uk/catalogue/PUB08694/ifs-uk-2010-chap2-inc-prev-dur.pdf (accessed on 9 December 2016).

Millsted, Rachel, and Hannah Frith. 2003. Being large-breasted: Women negotiating embodiment. Women's Studies International Forum 26: 455-65. [CrossRef]

Murphy, Elizabeth. 1999. 'Breast is best': Infant feeding decisions and maternal deviance. Sociology of Health \& Illness 21: 187-208.

Murphy, Elizabeth. 2003. Expertise and forms of knowledge in the government of families. The Sociological Review 51: 433-62. [CrossRef]

Murphy, Elizabeth, Susan Parker, and Christine Phipps. 1998. Food choice for babies. In The Nation's Diet: The Social Science of Food Choice. Edited by Anne Murcott. London: Longman, pp. 250-66.

NHS. 2016. Why Breastfeed?-Pregnancy and Baby Guide. NHS Choices. Available online: http://www.nhs.uk/ Conditions/pregnancy-and-baby/pages/why-breastfeed.aspx\#close (accessed on 9 December 2016).

Rippeyoung, Phyllis L. F. 2009. Feeding the state: Breastfeeding and women's well-being in context. Journal for the Association for Research on Mothering 11: 36-48.

Rippeyoung, Phyllis L. F., and Mary C. Noonan. 2012. Is breastfeeding truly cost free? Income consequences of breastfeeding for women. American Sociological Review 77: 244-67. [CrossRef]

Smyth, Lisa. 2008. Gendered spaces and intimate citizenship: The case of breastfeeding. European Journal of Women's Studies 15: 83-99. [CrossRef]

Taylor, Erin N., and Lora Ebert. 2012. For shame: Feminism, breastfeeding advocacy, and maternal guilt. Hypatia 27: 76-98. [CrossRef]

Thomson, Rachel. 2008. Thinking intergenerationally about motherhood. Studies in the Maternal 1: 1-24. [CrossRef]

Trickey, Heather, and Mary Newburn. 2012. Goals, dilemmas and assumptions in infant feeding education and support. Applying theory of constraints thinking tools to develop new priorities for action. Maternal $\mathcal{E}$ Child Nutrition 10: 72-91.

Wall, Glenda. 2001. Moral constructions of motherhood in breastfeeding discourse. Gender and Society 15: 592-610. [CrossRef]

Wolf, Joan. 2007. Is breast really best? Risk and total motherhood in the national breastfeeding awareness campaign. Journal of Health Politics, Policy and Law 32: 595-636. [CrossRef] [PubMed]

Wolf, Joan. 2011. Is Breast Best? New York: New York University Press.

(C) 2017 by the author. Licensee MDPI, Basel, Switzerland. This article is an open access article distributed under the terms and conditions of the Creative Commons Attribution (CC BY) license (http:/ / creativecommons.org/licenses/by/4.0/). 